3. One anonymous reviewer noted that we may have incorporated a potential confound into our procedures. Specifically, the brief exposure conditions involved shorter overall trial length than did the long preexposure conditions. This raises the possibility that duration of the trial per se rather than duration of the type of exposure (as we posit) was the critical variable. However, we are unable to reconcile the obtained pattern of results with the effect of a nonspecific "trials' length" factor. Remember that for each preexposure duration, the subject was tested twice-once with each of the two possible unambiguous configurations of the ambiguous figure (i.e., the two configurations were counterbalanced at each duration). Hence, to produce the obtained results, this trials' length factor would have to have been sensitive to the type of configuration presented, biasing the subject differently for the two configurations. This seems highly unlikely.

(Manuscript received August 26, 1991; revision accepted for publication May 19, 1992.)

\title{
Fifth International Conference on Vision in Vehicles University of Glasgow, Scotland September 9-11, 1993
}

The Applied Vision Association in conjunction with the Ergonomics Society and the Association of Optometrists announce the Fifth International Conference on Vision in Vehicles. This will be held at the Kelvin Conference Centre, University of Glasgow, Scotland, September 9-11, 1993. This multidisciplinary conference will provide an international forum for the exchange of information on current research encompassing all aspects of vision and its relationship to vehicle design. This includes both the internal and external design of the vehicle as well as the perceptual and cognitive capabilities and limitations of vehicle controller.

The scope of the conference will cover all types of vehicles where the task involves monitoring both in-vehicle displays and the surrounding visual environment in both day and night-time conditions. The conference will therefore be of interest to vehicle and instrument designers, psychologists, ergonomists, ophthalmologists, ophthalmic opticians, optometrists, highway engineers, traffic planners, lighting engineers, legislators, and anyone concerned with safety in vehicles.

Further information about the conference can be obtained from: VIV5, Academic Radiology, University Hospital, Queens Medical Centre, Nottingham NG7 2UH, U.K. (telephone, 44-0-602-709442; Fax, 44-0-602-709140). 